\title{
On soft/hard handoff for packet data services in cellular CDMA mobile systems
}

\author{
Ramon Ferrús, Pilar Díaz \\ Department of Signal Theory and Communications (UPC) \\ C/ Jordi Girona 1-3 Campus Nord-Edifici D4 08034 Barcelona-Spain \\ Phone: +34-3-401 5948 Fax: +34-3-401 7200 \\ e-mail: pilar, ferrus (a)xaloc.upc.es
}

\begin{abstract}
Benefits of macrodiversity operation for packet data services in third generation mobile systems are not obvious. Retransmission procedures to enhance link performance and higher downlink bandwidth requirements could question macrodiversity usage. This paper describes a simple methodology to compare soft and hard handoff performance in terms of transmission delay for packet data services. The handover procedures are based exclusively on power criteria and hysteresis margins.
\end{abstract}

\section{Introduction}

A wide area of research in cellular systems is related to handover issues. Regarding radio transmission layer, one important feature of CDMA-based systems is the ability of supporting soft handover, mechanism that allows the mobile to be connected simultaneously through the old and the target base stations while handover is performed. Instead, in a hard handover procedure, the old link is released before the new one is established. Macrodiversity could be seen as a generalisation of soft handover where the mobile could be connected to multiple base stations under certain conditions, without leading necessarily to a cell change.

Advantages of soft over hard handoff are mainly due to the inherent diversity gain that leads to improved power usage. In the reverse link, Selection Diversity (SD) based soft handover has been shown to increase system capacity [1]. In case of Maximal Ratio Combining (MRC), capacity bounds similar to those achieved in an isolated cell could be theoretically reached [2]. In [3], capacity increase due to SD soft handover is estimated about 1.1-1.4 times better than hard handoff results with 3-6 dB margins. Instead, in the forward link, results are less straightforward. Power reduction achieved by MRC combining does not directly yield capacity improvement because multiple base stations are assigning resources to the same mobile station [4]. In [5] it is shown that normal hard handoff $(3-6 \mathrm{~dB})$ results in capacity losses around 0.6-2.2 $\mathrm{dB}$ from optimal operation, achieved for soft handoff with low margins $(1-2 \mathrm{~dB})$ in a power controlled link. Such a better power usage could also be addressed as quality-of-service (QoS) improvement and cell coverage area increase in case of non-capacity limited systems. But benefits derived from macrodiversity adoption need to be contrasted with complexity added to mobile access networks.

Best effort or unconstrained delay data (UDD) services could achieve a required QoS by means of automatic-repeat-request (ARQ) procedures. Besides, asymmetrical applications with higher bandwidth requirements in the forward channel could question macrodiversity usage. Instead, macrodiversity seems to be justified for speech or delay constrained packet services for which QoS relies more on forward error correction (FEC) techniques.

This paper describes a simple methodology to compare soft and hard handoff performance in terms of transmission delay for packet data services and provides some repercussions of macrodiversity usage in mobile access networks mainly for packet data schemes. The paper is organised as follows. Next section deals with packet data transmission schemes in WCDMA and provides a general vision of macrodiversity repercussions in access networks. Section III describes the hard and soft handoff models based on power criteria and hysteresis margins. In section IV, the overall system model is explained and an analytical methodology to estimate transmission delay penalty for packet services is addressed. Section V reports some results and finally some conclusions are drawn.

\section{Macrodiversity and Packet Services}

Supporting of macrodiversity in mobile access networks impacts directly in their architecture. Figure 1 depicts the Radio Network Subsystem (RNS) portion of the UTRAN proposal [6]. According to ETSI proposal, but basically extensible to most proposals, access networks will consist of controllers connected to a Core Network, known as Radio Network Controllers (RNCs), and cell sites or base stations hanging from those controllers, known as Node-Bs. 
Soft handover operation is envisaged for cells using physical layer FDD mode (WCDMA) and identical frequency assignment. If candidate cells belong to different Node-B, macrodiversity combining and multicasting needs to be performed at RNC. Softer handover occurs. when related cells belong to the same Node B. In that case, MRC combining could be used at Node-B in the uplink while, in the former, frame selection combining is performed at RNC. Thus, stream interfaces between Node-B and RNC and inter-RNC have to cope with the transport of user data frame blocks but also synchronisation and quality information multiplexed onto the same transport mechanism. The best-suited candidate transport technology seems to be AAL2 over ATM. Furthermore; the placement of functions related to Medium Access Control (MAC) and Radio Link Control (RLC) is also conditioned. As multicasting or combining is performed at physical layer, that layer should be extended up to the RNC, and consequently MAC and RLC functions need to be located at RNC if macrodiversity is allowed.

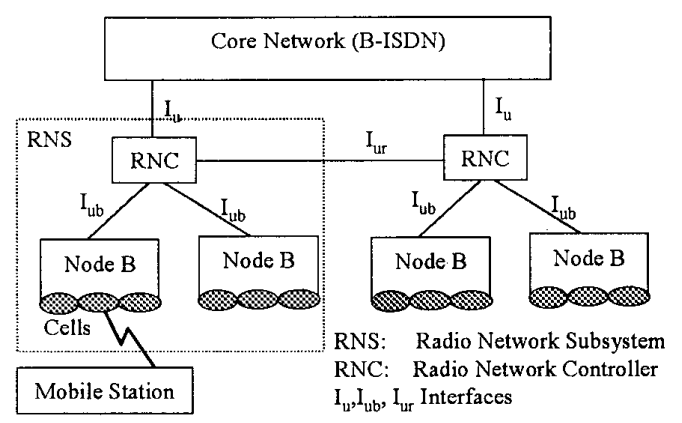

Figure 1. UTRAN proposed architecture.

Packet access in WCDMA is under study. In [6] a dual approach is outlined in which short infrequent packets are transmitted in the random access channel while larger packets use dedicated channels for the transmission of a single packet or a sequence of them. Handover in common channel transmission is reduced to a cell re-selection mechanism, while typical handover procedures only seem to apply to dedicated channels. However, in [7] a higher capacity common packet channel is introduced. This packet bearer is presented for UDD services from $8 \mathrm{kbits} / \mathrm{s}$ up to $2 \mathrm{Mbps}$. In such case, macrodiversity implementation in front of cell re-selection could lead to considerable interference reduction. But macrodiversity usage when some kind of scheduling is needed results in a complex MAC mechanism since co-ordination among accesses through multiple base stations is needed. The same problem appears in dedicated mode with scheduling. Difficulty accentuates even more when different RNC are involved.

Soft handoff results in truly seamless handover without any disruption of service. Instead, hard handoff could originate relatively short physical layer breaks. However, this service interruption does not avoid the possibility of carrying out lossless handoffs for data services since link layer mechanisms could be used to mitigate it.

\section{Modelling Soft and Hard Handoff}

Hard handoff model used by Viterbi et al. in [1] to assess capacity performance could be shown to result in conservative estimation in front of soft handoff since distance criterion is used to select the active base station for each mobile. The hard handoff mechanism considered in the current work has already been detailed in [3,5] for uplink and downlink channels and is exclusively based on power criteria.

Hard handoff is modelled by a hysteresis margin denoted as $\Delta_{\mathrm{HHO}}$. If power from a neighbouring base station goes above the current link by at least $\Delta_{\mathrm{HHO}} \mathrm{dB}$ a handoff request is issued. The current base station is chosen randomly among those received above the best power level minus the hysteresis margin. The soft handoff procedure might be also characterised by a power margin denoted as $\Delta_{\mathrm{SHO}}$. The meaning of this margin depends on the considered channel. $\Delta_{\text {SHO }}$ will be used to determine which base stations are going to assign resources to a mobile terminal in the downlink. In the uplink, $\Delta_{\mathrm{SHO}}$ will be used to determine which base stations demodulate signal from a mobile and forward it to the macrodiversity combiner.

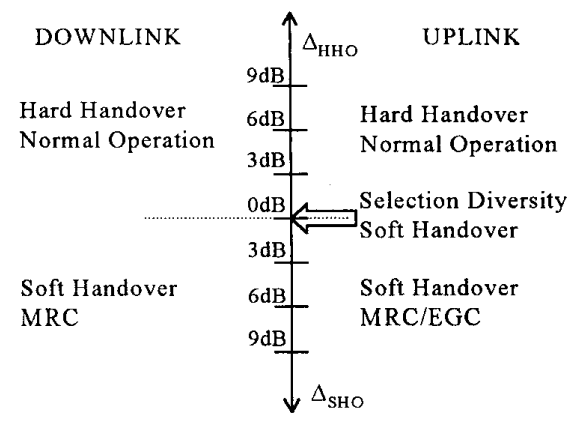

Figure 2. $\Delta_{\mathrm{HHO}}$ and $\Delta_{\mathrm{SHO}}$ allow establishing a gradual transition between hard and soft handoff.

\section{System Model}

Let us assume a macrocell scenario of regular hexagonal-shaped cells. A static approach analysis has been adopted to assess system performance in 
order to avoid long time consuming simulations. Firstly, uplink interference and downlink power allocation conditions are gathered by means of Monte Carlo simulations for Ns simultaneous transmitting/receiving users per cell. Users are supposed to be uniformly distributed within the total service area. Statistics characterisation is performed from the collected information using MLE estimation. The following sections briefly outlines uplink interference characterisation and power allocation in the downlink although a detailed analysis can be found in [3,5]. Particularly in [3], combining techniques as SD, MRC and EGC for the uplink channels are discussed. In [5], downlink performance is addressed for power/nonpower controlled links and different power allocation strategies.

To calculate the received power, the propagation model used consists of the product of the distance to the $\mu^{\text {th }}$ power and a lognormal component $\xi$ accounting for shadowing losses. Rayleigh fading components are not included since they are supposed to be computed in the required bit energyto-interference ratio.

\section{A. Downlink characterisation}

The received $\mathrm{E}_{\mathrm{b}} / \mathrm{N}_{0}$ for a mobile terminal $i$ connected through base station BS $j$ could be expressed by

$$
\left(\frac{E_{b}}{N_{o}}\right)_{i}=G_{p} \frac{\beta_{j} \phi_{j}^{i} S_{j}^{i}}{\sum_{t=1}^{K} S_{t}^{i}-\delta \cdot S_{j}^{i}+\eta}
$$

where $\beta_{\mathrm{j}}$ is the fraction of the total power from base station $j$ devoted to subscribers, $\phi_{j}{ }^{i}$ the percentage of $\beta_{\mathrm{j}}$ allocated to the mobile $i, \mathrm{~S}_{\mathrm{t}}$ refers to the total power received from base station $t, \mathrm{G}_{\mathrm{p}}$ is the processing gain and $\eta$ represents the background noise. As spreading sequences in the forward channel could provide orthogonality between different user channels, a factor denoted by $\delta$ is introduced to account for the percentage of interference reduction due to orthogonality.

When macrodiversity is applied and a coherent RAKE diversity combiner is used at the mobile receiver, the resulting $E_{b} / N_{0}$ could be estimated as the sum of individual signal-to-interference ratios in each active finger in the RAKE demodulator. In this case (1) could be rewritten as

$$
\left(\frac{E_{b}}{N_{o}}\right)_{i, \text { total }}=\sum_{j=1}^{m}\left(\frac{E_{b}}{N_{o}}\right)_{i, j}=G_{p} \sum_{j=1}^{m_{j}} \frac{\beta_{j} \phi_{j}^{i} S_{j}^{i}}{\sum_{t=1}^{K} S_{t}^{i}-\delta \cdot S_{j}^{i}+\eta}
$$

where $m_{j}$ is the number of base stations assigning power to subscriber $i$.
Assuming all base station within the Active Set allocate identical fraction of power, the minimum $\phi^{i}$ can be calculated. Normalising $\phi^{i}$ by the system dependent factors and denoting the new parameter as $\Phi^{\mathrm{i}}$, the maximum power a base station could allocate is restricted by

$$
\Phi \equiv \sum_{i=1}^{M} \Phi^{i} \leq \frac{\beta G_{p}}{\left(E_{b} / N_{o}\right)_{i, r e q}}=C
$$

where $\mathbf{M}$ is the number of users simultaneously served by a single BS. Considering $M$ large enough to apply the Central Limit Theorem, $\Phi$ could be approximated by a Gaussian random variable.

\section{B. Uplink characterisation}

For the uplink channel, the experimented $\mathrm{E}_{\mathrm{b}} / \mathrm{N}_{0}$ for mobile $i$ at could be written as

$$
\left(\frac{E_{b}}{N_{o}}\right)_{i} \approx G_{p} \frac{S^{i}}{\sum_{\substack{j=1 \\ j \neq i}}^{M} S^{j}\left(\frac{r_{t j}}{r_{o j}}\right)^{\mu} 10^{\frac{\zeta_{o j}-\zeta_{i j}}{10}}+\eta}
$$

where $t$ refers to the different base stations each mobile $i$ could be linked to and $\mathrm{S}^{\mathrm{j}}$ models the target received power. Equivalently to (3), we have for the uplink channel the following bound

$$
I_{T}=\sum_{\substack{j=1 \\ j \neq i}}^{M} \frac{S^{j}}{S^{i}}\left(\frac{r_{t j}}{r_{o j}}\right)^{\mu} 10^{\frac{\zeta_{o j}-\zeta_{t j}}{10}} \leq \frac{G_{p}}{\left(\frac{E_{b}}{N_{o}}\right)_{r e q}}-\frac{\eta}{S^{i}}=\delta
$$

Resorting again to the Central Limit Theorem, $\mathbf{I}_{\mathrm{T}}$ could be modelled as a Gaussian random variable. The fairness of such approximations will depend on how large is $\mathbf{M}$ but, as we are interested in system capacity values, this condition will be normally held.

\section{Probability Density Function of $\mathbf{E}_{b} / \mathbf{N}_{0}$}

Once statistics for uplink interference $\left(\mathrm{I}_{\mathrm{T}}\right)$ and downlink power assignment (Ф) have been collected, the current $E_{b} / N_{0}$ experimented in each link could be obtained as

$$
\begin{aligned}
& \left(\frac{E_{b}}{N_{o}}\right)^{u l}=\min \left(\left(\frac{E_{b}}{N_{o}}\right)_{r e q}^{u l}, \frac{G_{p}}{I_{T}}\right) \\
& \left(\frac{E_{b}}{N_{o}}\right)^{d l}=\min \left(\left(\frac{E_{b}}{N_{o}}\right)_{r e q}^{d l}, \frac{G_{p}}{\Phi}\right)
\end{aligned}
$$

Assuming $\mathrm{I}_{\mathrm{T}}$ and $\Phi$ normal distributed, the probability density function of the $E_{b} / N_{0}$ can be written as 


$$
\begin{aligned}
& \int \quad \begin{array}{lll}
0 & E_{b} / N_{o}>\left(\frac{E_{b}}{N_{o}}\right)_{\text {req }}
\end{array}
\end{aligned}
$$

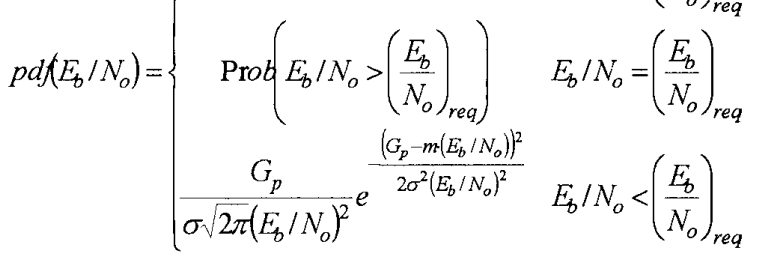

(7) where $m$ and $\sigma$ are derived from

Uplink $\left\{\begin{array}{l}m=\operatorname{mean}\left(I_{T}\right)=N_{s} \operatorname{mean}(\psi) \\ \sigma=\operatorname{std}\left(I_{T}\right)=\sqrt{N_{s}} \operatorname{std}(\psi)\end{array}\right.$

Downlink $\left\{\begin{array}{l}m=\operatorname{mean}(\Phi)=N_{s} \operatorname{mean}(\bar{\Phi}) \\ \sigma=\operatorname{std}(\Phi)=\sqrt{N_{s}} \operatorname{std}(\bar{\Phi})\end{array}\right.$

where $\psi$ and $\bar{\Phi}$ have been found to untie $\mathrm{N}_{\mathrm{s}}$ from propagation issues and adopted handover mechanism [3,5].

\section{Delay Estimation}

Data packets to be transmitted are passed to the RLC layer. At that point, packets are segmented into radio blocks (RLC blocks) and a number of them transmitted each $10 \mathrm{~ms}$ frame depending on channel bit rate. Total delay until complete reception of the entire packet at the other extreme is due to medium access control operation and transmission delay. Assuming MAC performance independent from the handover mechanism adopted, only transmission delay will be taken into account for comparison purposes.

The correct reception of RLC blocks at each frame depends on carrier-to-interference ratio assured during that period. Thus, the RLC block error rate (BLER) could be estimated from link level simulations versus $\mathrm{E}_{\mathrm{b}} / \mathrm{N}_{0}$ conditions. If a Selective Repeat ARQ is used at RLC layer and return channel is assumed error free, the mean and standard deviation of the transmission delay can be given as

$$
\operatorname{mean}(\text { Delay })=\Delta=\frac{1}{1-B L E R\left(E_{b} N_{o}\right)}
$$

If perfect power control is considered in both links, whenever interference level is below the theoretical limit given by (5) in the uplink or the base station is able to assign the required power to each mobile terminal in the downlink (3), the $\left(\mathrm{E}_{\mathrm{b}} / \mathrm{No}\right)_{\text {req }}$ will be guaranteed. The choice of the required $\mathrm{E}_{\mathrm{b}} / \mathrm{N}_{0}$ needs to be optimised to maximise system capacity. In [8] a methodology based only on link level simulations is suggested to estimate the optimum $\mathrm{E}_{\mathrm{b}} / \mathrm{N}_{0}$ target.
Once the optimum operation condition is obtained, the transmission delay normalised to the delay at optimum capacity condition $\Delta_{\text {req }}$ is estimated as

$$
\begin{aligned}
& \bar{\Delta}=\frac{\Delta}{\Delta_{\text {req }}}=\operatorname{Prob}\left(E_{b} / N_{o} \geq\left(E_{b} / N_{o}\right)_{\text {req }}\right) \\
& +\int_{0}^{\left(E_{b} / N_{o}\right)_{\text {req }}} \frac{1-B L E R\left(\left(E_{b} / N_{o}\right)_{\text {req }}\right)}{1-B L E A\left(E_{b} / N_{o}\right)} f d p\left(E_{b} / N_{o}\right) \cdot d E_{b} / N_{o}
\end{aligned}
$$

\section{Results}

Table I shows estimated mean and standard deviation of $\bar{\Phi}$ for different handover margins. Values within brackets refer to the $95 \%$ confidence interval obtained by MLE estimation. A path slope $\mu=4$, shadowing deviation of $4 \mathrm{~dB}$ and $50 \%$ correlated losses have been assumed. Orthogonality factor has been set to 0.5 and background noise is $20 \mathrm{~dB}$ below received power. Similar tables are available in [3] for the uplink channel.

Table I. Statistics of downlink power allocation

\begin{tabular}{|c|c|c|c|c|}
\cline { 2 - 5 } \multicolumn{1}{c|}{} & \multicolumn{2}{c|}{ Hard Handover } & \multicolumn{2}{c|}{ Sof Handover } \\
\hline $\mathrm{N}_{\text {Ho }}$ & Mean & $\begin{array}{c}\text { Standard } \\
\text { Deviation }\end{array}$ & Mean & $\begin{array}{c}\text { Standard } \\
\text { Deviation }\end{array}$ \\
\hline 0 & 0.96 & $\begin{array}{c}0.82 \\
(0.79 / 0.86)\end{array}$ & $\begin{array}{c}0.96 \\
(0.95 / 0.98)\end{array}$ & $\begin{array}{c}0.82 \\
(0.79 / 0.86)\end{array}$ \\
\hline 1 & 0.98 & 0.87 & 0.97 & 0.73 \\
& $(0.97 / 1.00)$ & $(0.84 / 0.91)$ & $(0.97 / 0.99)$ & $(0.70 / 0.76)$ \\
\hline 2 & 1.01 & 0.95 & 1.00 & 0.69 \\
& $(1.00 / 1.03)$ & $(0.91 / 0.99)$ & $(1.00 / 1.02)$ & $(0.66 / 0.72)$ \\
\hline 3 & 1.06 & 1.07 & 1.04 & 0.66 \\
& $(1.06 / 1.07)$ & $(1.06 / 1.09)$ & $(1.03 / 1.04)$ & $(0.65 / 0.67)$ \\
\hline 6 & 1.42 & 1.95 & 1.19 & 0.70 \\
& $(1.42 / 1.43)$ & $(1.92 / 1.97)$ & $(1.19 / 1.20)$ & $(0.69 / 0.71)$ \\
\hline 9 & 2.32 & 3.78 & 1.38 & 0.76 \\
& $(2.31 / 2.34)$ & $(3.73 / 3.83)$ & $(1.38 / 1.38)$ & $(0.75 / 0.77)$ \\
\hline
\end{tabular}

Delay penalty has been obtained for a single 4096Mchips/s carrier with $240 \mathrm{kbits} / \mathrm{s}$ radio bearers. An effective processing gain of $12.3 \mathrm{~dB}$ has been considered and link level simulations reported in [6] for Outdoor to Indoor and Pedestrian A with antennae diversity and Indoor office A have been used for uplink and downlink channels respectively.

Figure 3 and 4 show downlink performance for $50 \%$ and perfect orthogonality factors respectively. Soft handover with reduced margins (1-2) performs the best. For $50 \%$ orthogonality factor, a hard handover procedure of $3 \mathrm{~dB}$ could support up to 7 simultaneous users with delay penalty below $20 \%$. For $1 \mathrm{~dB}$ soft handoff the number of users increases up to 9 . Higher hard handoff margins $(6 \mathrm{~dB})$ folds the transmission delay. Figure 5 depicts results for the uplink channel characterised with the same propagation conditions as the downlink. It could be seen that results are similar in the hard handoff 
region. Low hard handover margin $(3 \mathrm{~dB})$ derives in a slight increase of transmission delay compared to a SD mechanism. However, MRC combining outperforms SD results and could tolerate up to 13 mobiles with delay worsening below $20 \%$.

\section{Conclusions}

Some implications macrodiversity has on transport layer within future mobile access networks and aspects related to MAC and RLC functions for packet bearers have been addressed. Basically, increased complexity and limitations introduced to support macrodiversity need to be balanced with capacity or QoS improvement.

To that end, a methodology to calculate an estimation of transmission delay penalty for uplink and downlink channel has been proposed. Results could be obtained from link level simulations and uplink interference and downlink power allocation statistics without long time consuming simulations.

\section{Acknowledgement}

This work has been performed in the framework of the CICYT project TIC98-0684.

\section{References}

[1] J. Viterbi et alt. "Soft Handoffs Extends CDMA Cell Coverage and Increases Reverse Link Capacity" IEEE JSAC, vol. 12, $\mathrm{n}^{\circ} 8$, October 1994.

[2] S.V. Hanly, "Capacity and power control in spread spectrum macrodiversity radio networks", IEEE Trans. on Com., vol. 44,Feb 1996.

[3] R. Ferrus and P. Díaz, "On hard/soft handoff and macrodiversity in a CDMA mobile system", to appear in proc. of PIMRC'99.

[4] Chin-Chun Lee and Raymond Steele, "Effects of Soft and Softer Handoffs on CDMA System Capacity", IEEE Trans on Veh. Tech, vol. 47, $\mathrm{n}^{\circ} 3$, August 1998.

[5] R Ferrus and P.Diaz, "On macrodiversity and power control for the forward link in a CDMA mobile system", AMOS'99

[6] The ETSI UMTS Terrestrial Radio Access (UTRA) ITU-R RTT, June 1998.

[7] Wideband Packet CDMA (WP-CDMA) ITU-R RTT Candidate Submission, June 1998.

[8] B. Christer V. Johansson, "Packet Data Capacity in a Wideband CDMA System", VTC'98.

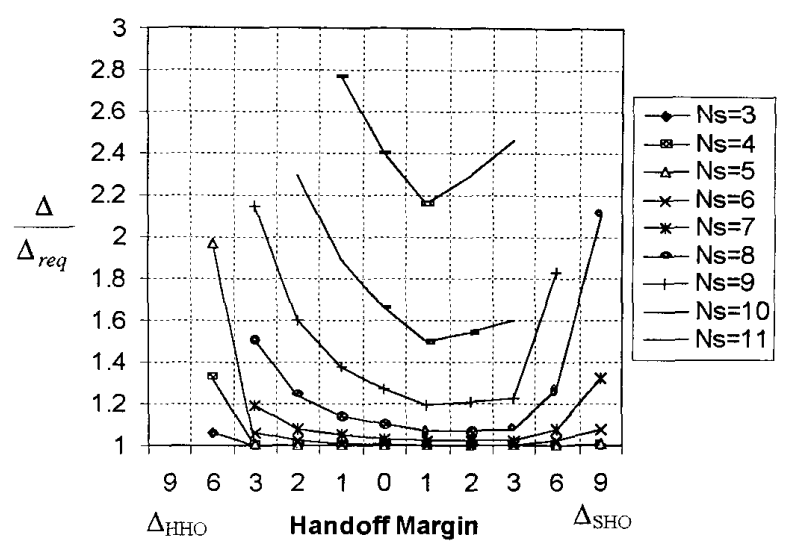

Figure 3. Downlink channel with $50 \%$ orthogonality factor.

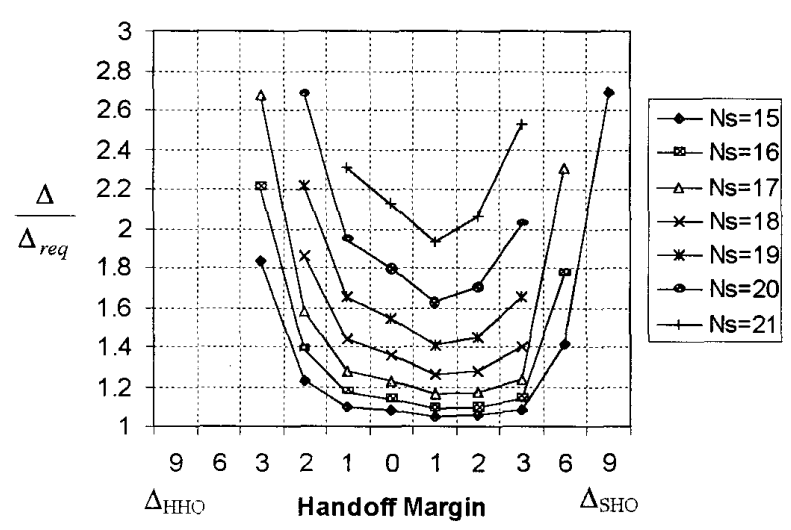

Figure 4. Downlink channel with perfect orthogonality factor

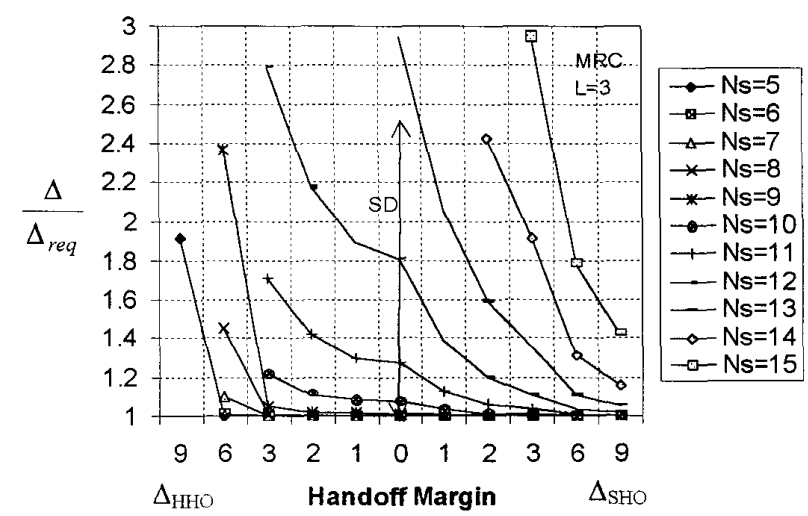

Figure 5. Uplink channel. MRC combining is limited to $\mathrm{L}=3$ branches. 